\title{
Boundary value problems for a new class of three-point nonlocal Riemann-Liouville integral boundary conditions
}

\author{
Jessada Tariboon ${ }^{1 *}$, Thanin Sitthiwirattham ${ }^{1}$ and Sotiris K Ntouyas ${ }^{2}$
}

*Correspondence:

jessadat@kmutnb.ac.th

${ }^{1}$ Department of Mathematics,

Faculty of Applied Science, King

Mongkut's University of Technology

North Bangkok, Bangkok, 10800,

Thailand

Full list of author information is

available at the end of the article

\begin{abstract}
In this paper we investigate the existence and uniqueness of solutions for a new class of fractional boundary value problems involving three-point nonlocal

Riemann-Liouville integral boundary conditions. Some new results are obtained by using fixed point theorems. Illustrative examples of our results are also presented.

MSC: $26 \mathrm{~A} 33 ; 34 \mathrm{~A} 08$
\end{abstract}

Keywords: fractional differential equations; nonlocal boundary conditions; fixed point theorems

\section{Introduction}

Fractional order differential equations have been of great interest recently because they play a vital role in describing many phenomena related to physics, chemistry, mechanics, control systems, flow in porous media, electrical networks, mathematical biology and viscoelasticity. For a reader interested in the systematic development of the topic, we refer to the books [1-7]. A variety of results on initial and boundary value problems of fractional differential equations and inclusions can easily be found in the literature on the topic. For some recent results, we can refer to [8-19] and references cited therein.

Bai [11] discussed the existence of positive solutions for the following three-point fractional boundary value problem:

$$
\begin{aligned}
& D^{q} u(t)=f(t, u(t)), \quad 1<q \leq 2, t \in(0,1), \\
& u(0)=0, \quad u(1)=\alpha \beta u(\eta), \quad 0<\eta<1,
\end{aligned}
$$

where $D^{q}$ denotes the Riemann-Liouville fractional derivative, and $0<\beta \eta^{q-1}<1$. Some existence results for at least one positive solution are obtained by the use of fixed point index theory.

In paper [12] the authors studied the following boundary value problem of fractional order differential equations with three-point fractional integral boundary conditions:

$$
\begin{aligned}
& { }^{c} D^{q} u(t)=f(t, u(t)), \quad 1<q \leq 2, t \in(0,1), \\
& u(0)=0, \quad u(1)=\alpha I^{p} u(\eta), \quad 0<\eta<1,
\end{aligned}
$$

(c) 2013 Tariboon et al.; licensee Springer. This is an Open Access article distributed under the terms of the Creative Commons Attribution License (http://creativecommons.org/licenses/by/2.0), which permits unrestricted use, distribution, and reproduction in any medium, provided the original work is properly cited. 
where ${ }^{c} D^{q}$ denotes the Caputo fractional derivative of order $q, I^{p}$ is the Riemann-Liouville fractional integral of order $p, f \in C([0,1] \times \mathbb{R})$ and $\alpha \in \mathbb{R}, \alpha \neq \Gamma(p+2) / \eta^{p+1}$. Existence and uniqueness results are proved via Banach's contraction principle and Schaefer's fixed point theorem. The results of [12] are completed in [13], by existence results via Krasnoselskii's fixed point theorem and Leray-Schauder's degree theory, and extended to cover the multivalued case.

In [14] existence and uniqueness results are obtained for a single and multivalued case for the following boundary value problem of fractional order differential equations with nonlocal and fractional integral boundary conditions:

$$
\begin{array}{ll}
D^{q} u(t)=f(t, u(t)), & 1<q \leq 2, t \in(0,1), \\
u(0)=u_{0}+g(u), & u(1)=\alpha I^{\alpha} u(\eta), \quad 0<\eta<1,
\end{array}
$$

where $g: C([0,1], \mathbb{R}) \rightarrow \mathbb{R}$.

Recently, in [20] the following boundary value problem of fractional differential equations with a fractional integral condition:

$$
\left\{\begin{array}{l}
D^{q} u(t)=f\left(t, u(t), D^{p} u(t)\right), \quad 0<t<1,1<q \leq 2,0<p<1 \\
u(0)=0, \quad u^{\prime}(1)=\alpha I^{p} u(1)
\end{array}\right.
$$

was studied. Existence and uniqueness results are proved via Banach's contraction principle and Leray-Schauder's nonlinear alternative.

In [16] existence and uniqueness results are investigated for the following boundary value problem of fractional order differential equations with four-point nonlocal Riemann-Liouville fractional integral boundary conditions:

$$
\begin{aligned}
& D^{q} u(t)=f(t, u(t)), \quad 1<q \leq 2, t \in(0,1), \\
& u(0)=a I^{\beta} u(\eta), \quad u(1)=b I^{\alpha} u(\sigma),
\end{aligned}
$$

where $0<\alpha, \beta \leq 1$ and $0<\eta, \sigma<1$.

In this paper, we study a new class of three-point boundary value problems of fractional order differential equations with nonlocal Riemann-Liouville fractional integral boundary conditions. More precisely, we consider the nonlinear fractional differential equation

$$
D^{\alpha} u(t)=f(t, u(t)), \quad 1<\alpha \leq 2,0<t<T,
$$

subject to nonlocal fractional integral conditions

$$
u(\eta)=0, \quad I^{v} u(T) \equiv \int_{0}^{T} \frac{(T-s)^{v-1}}{\Gamma(v)} u(s) d s=0,
$$

where $\eta \in(0, T)$ is a given constant. The novelty of this boundary value problem lies in the fact that instead of the value $u(0)$, which appeared in all the above mentioned boundary value problems, we have the value $u(\eta)$ for some $\eta \in(0, T)$.

The paper is organized as follows. In Section 2 we recall some preliminary facts that we need in the sequel. In Section 3 we prove our main results. Some examples to illustrate our results are presented in Section 4. 


\section{Preliminaries}

In this section we introduce some notations, definitions of fractional calculus $[3,4]$ and present preliminary results needed in our proofs later.

Definition 2.1 The Riemann-Liouville fractional integral of order $\alpha>0$ of a function $g \in$ $L^{1}((0, T), \mathbb{R})$ is defined by

$$
I^{\alpha} g(t)=\int_{0}^{t} \frac{(t-s)^{\alpha-1}}{\Gamma(\alpha)} g(s) d s
$$

where $\Gamma$ is the gamma function.

Definition 2.2 The Riemann-Liouville fractional derivative of order $\alpha>0$ of a continuous function $g:(0, \infty) \rightarrow \mathbb{R}$ is defined by

$$
D^{\alpha} g(t)=\frac{1}{\Gamma(n-\alpha)}\left(\frac{d}{d t}\right)^{n} \int_{0}^{t} \frac{g(s)}{(t-s)^{\alpha-n+1}} d s,
$$

where $n=[\alpha]+1,[\alpha]$ denotes the integral part of real number $\alpha$, provided the right-hand side is point-wise defined on $(0, \infty)$.

Lemma 2.1 (see [4]) Let $\alpha>0$ and $y \in C(0, T) \cap L(0, T)$. Then the fractional differential equation $D^{\alpha} y(t)=0$ has a unique solution

$$
y(t)=c_{1} t^{\alpha-1}+c_{2} t^{\alpha-2}+\cdots+c_{n} t^{\alpha-n},
$$

where $c_{i} \in \mathbb{R}, i=1,2, \ldots, n$ and $n=[\alpha]+1$.

Lemma 2.2 Suppose that $\eta \neq \frac{(\alpha-1) T}{v+\alpha-1}, 1<\alpha \leq 2, v>0$ and $h \in A C[0, T]$. Then the problem

$$
\begin{aligned}
& D^{\alpha} x(t)=h(t), \quad 0<t<T, \\
& x(\eta)=0, \quad I^{v} x(T)=0, \quad \eta \in(0, T),
\end{aligned}
$$

can be written as an integral equation

$$
\begin{aligned}
x(t)= & \int_{0}^{t} \frac{(t-s)^{\alpha-1}}{\Gamma(\alpha)} h(s) d s \\
& -\frac{(v+\alpha-1) t^{\alpha-1}-T(\alpha-1) t^{\alpha-2}}{(v+\alpha-1) \eta^{\alpha-2} \Gamma(\alpha) \Omega} \int_{0}^{\eta}(\eta-s)^{\alpha-1} h(s) d s \\
& +\frac{t^{\alpha-1}-\eta t^{\alpha-2}}{(v+\alpha-1) T^{v+\alpha-2} \Gamma(\alpha-1) \Omega} \int_{0}^{T}(T-s)^{v+\alpha-1} h(s) d s,
\end{aligned}
$$

where

$$
\Omega=\eta-\frac{(\alpha-1) T}{v+\alpha-1} .
$$


Proof From (2.1) and Lemma 2.1, we have

$$
x(t)=c_{1} t^{\alpha-1}+c_{2} t^{\alpha-2}+\int_{0}^{t} \frac{(t-s)^{\alpha-1}}{\Gamma(\alpha)} h(s) d s .
$$

The first condition of (2.2) implies

$$
c_{1} \eta^{\alpha-1}+c_{2} \eta^{\alpha-2}=-\int_{0}^{\eta} \frac{(\eta-s)^{\alpha-1}}{\Gamma(\alpha)} h(s) d s
$$

Using the Riemann-Liouville fractional integral of order $v>0$ to (2.5) and applying Dirichlet's formula [1, p.56], we obtain

$$
\begin{aligned}
I^{v} x(t)= & \frac{c_{1}}{\Gamma(v)} \int_{0}^{t}(t-s)^{\nu-1} s^{\alpha-1} d s+\frac{c_{2}}{\Gamma(v)} \int_{0}^{t}(t-s)^{v-1} s^{\alpha-2} d s \\
& +\frac{1}{\Gamma(v) \Gamma(\alpha)} \int_{0}^{t} \int_{0}^{s}(t-s)^{\nu-1}(s-\rho)^{\alpha-1} h(\rho) d \rho d s \\
= & c_{1} \frac{\Gamma(\alpha)}{\Gamma(v+\alpha)} t^{\nu+\alpha-1}+c_{2} \frac{\Gamma(\alpha-1)}{\Gamma(v+\alpha-1)} t^{\nu+\alpha-2} \\
& +\frac{1}{\Gamma(v+\alpha)} \int_{0}^{t}(t-s)^{v+\alpha-1} h(s) d s .
\end{aligned}
$$

The second condition of (2.2) implies

$$
c_{1} \frac{\Gamma(\alpha)}{\Gamma(\nu+\alpha)} T^{\nu+\alpha-1}+c_{2} \frac{\Gamma(\alpha-1)}{\Gamma(\nu+\alpha-1)} T^{\alpha+v-2}=-\int_{0}^{T} \frac{(T-s)^{\nu+\alpha-1}}{\Gamma(v+\alpha)} h(s) d s .
$$

Solving the linear equations (2.6)-(2.7) for unknown constants $c_{1}$ and $c_{2}$, we have

$$
\begin{aligned}
c_{1}= & -\frac{1}{\eta^{\alpha-2} \Gamma(\alpha) \Omega} \int_{0}^{\eta}(\eta-s)^{\alpha-1} h(s) d s \\
& +\frac{\Gamma(v+\alpha-1)}{T^{v+\alpha-2} \Gamma(\alpha-1) \Omega} \int_{0}^{T} \frac{(T-s)^{v+\alpha-1}}{\Gamma(v+\alpha)} h(s) d s
\end{aligned}
$$

and

$$
\begin{aligned}
c_{2}= & \frac{T(\alpha-1)}{(v+\alpha-1) \eta^{\alpha-2} \Gamma(\alpha) \Omega} \int_{0}^{\eta}(\eta-s)^{\alpha-1} h(s) d s \\
& -\frac{\eta \Gamma(\nu+\alpha-1)}{T^{v+\alpha-2} \Gamma(\alpha-1) \Omega} \int_{0}^{T} \frac{(T-s)^{\nu+\alpha-1}}{\Gamma(\nu+\alpha)} h(s) d s,
\end{aligned}
$$

where constant $\Omega$ is defined by (2.4). Substituting constants $c_{1}$ and $c_{2}$ in (2.5), we obtain (2.3).

Let $\mathcal{C}=C([0, T], \mathbb{R})$ denote the Banach space of all continuous functions from $[0, T]$ to $\mathbb{R}$ endowed with the norm defined by $\|u\|=\sup _{t \in[0, T]}|u(t)|$. As in Lemma 2.2, we define 
an operator $A: \mathcal{C} \rightarrow \mathcal{C}$ by

$$
\begin{aligned}
(A u)(t)= & \int_{0}^{t} \frac{(t-s)^{\alpha-1}}{\Gamma(\alpha)} f(s, u(s)) d s \\
& -\frac{(v+\alpha-1) t^{\alpha-1}-T(\alpha-1) t^{\alpha-2}}{(v+\alpha-1) \eta^{\alpha-2} \Gamma(\alpha) \Omega} \int_{0}^{\eta}(\eta-s)^{\alpha-1} f(s, u(s)) d s \\
& +\frac{t^{\alpha-1}-\eta t^{\alpha-2}}{(v+\alpha-1) T^{\nu+\alpha-2} \Gamma(\alpha-1) \Omega} \int_{0}^{T}(T-s)^{\nu+\alpha-1} f(s, u(s)) d s .
\end{aligned}
$$

It should be noticed that problem (1.6)-(1.7) has solutions if and only if the operator $A$ has fixed points.

\section{Main results}

We are in a position to establish our main results. In the following subsections, we prove existence as well as existence and uniqueness results for BVP (1.6)-(1.7) by using a variety of fixed point theorems.

\subsection{Existence and uniqueness results via Banach's fixed point theorem}

In this subsection we give first an existence and uniqueness result for BVP (1.6)-(1.7) by using Banach's fixed point theorem.

Theorem 3.1 Assume that

$\left(\mathrm{H}_{1}\right)$ there exists a constant $L>0$ such that $|f(t, u)-f(t, v)| \leq L|u-v|$ for each $t \in[0, T]$ and $u, v \in \mathbb{R}$.

If

$$
\begin{aligned}
\Lambda:= & L T^{\alpha}\left\{\frac{1}{\Gamma(\alpha+1)}+\frac{\eta^{2}(v+2(\alpha-1))}{(v+\alpha-1) T \Gamma(\alpha+1)|\Omega|}\right. \\
& \left.+\frac{T+\eta}{(v+\alpha-1)(v+\alpha) \Gamma(\alpha-1)|\Omega|}\right\}<1,
\end{aligned}
$$

then problem (1.6)-(1.7) has a unique solution in $[0, T]$.

Proof We transform problem (1.6)-(1.7) into a fixed point problem, $u=A u$, where the operator $A$ is defined by (2.8). Obviously, fixed points of the operator $A$ are solutions of problem (1.6)-(1.7). Using the Banach contraction principle, we shall show that $A$ has a fixed point.

Setting $\sup _{t \in[0, T]}|f(t, 0)|=M<\infty$ and choosing $r \geq \frac{\Lambda M}{(1-\Lambda) L}$, we show that $A B_{r} \subset B_{r}$, where $B_{r}=\{x \in \mathcal{C}:\|x\| \leq r\}$. For $x \in B_{r}$, we have

$$
\begin{aligned}
\|A u\| \leq & \sup _{t \in[0, T]}\left\{\int_{0}^{t} \frac{(t-s)^{\alpha-1}}{\Gamma(\alpha)}|f(s, u(s))| d s\right. \\
& +\frac{T^{\alpha-1}(v+2(\alpha-1))}{(v+\alpha-1) \eta^{\alpha-2} \Gamma(\alpha)|\Omega|} \int_{0}^{\eta}(\eta-s)^{\alpha-1}|f(s, u(s))| d s \\
& \left.+\frac{T^{\alpha-2}(T+\eta)}{(v+\alpha-1) T^{v+\alpha-2} \Gamma(\alpha-1)|\Omega|} \int_{0}^{T}(T-s)^{\nu+\alpha-1}|f(s, u(s))| d s\right\}
\end{aligned}
$$




$$
\begin{aligned}
\leq & \sup _{t \in[0, T]}\left\{\int_{0}^{t} \frac{(t-s)^{\alpha-1}}{\Gamma(\alpha)}(|f(s, u(s))-f(s, 0)|+|f(s, 0)|) d s\right. \\
& +\frac{T^{\alpha-1}(v+2(\alpha-1))}{(v+\alpha-1) \eta^{\alpha-2} \Gamma(\alpha)|\Omega|} \int_{0}^{\eta}(\eta-s)^{\alpha-1}(|f(s, u(s))-f(s, 0)|+|f(s, 0)|) d s \\
& +\frac{T+\eta}{(v+\alpha-1) T^{\nu} \Gamma(\alpha-1)|\Omega|} \\
& \left.\times \int_{0}^{T}(T-s)^{v+\alpha-1}(|f(s, u(s))-f(s, 0)|+|f(s, 0)|) d s\right\} \\
\leq & (L r+M) \sup _{t \in[0, T]}\left\{\frac{1}{\Gamma(\alpha)} \int_{0}^{t}(t-s)^{\alpha-1} d s\right. \\
& +\frac{T^{\alpha-1}(v+2(\alpha-1))}{(v+\alpha-1) \eta^{\alpha-2} \Gamma(\alpha)|\Omega|} \int_{0}^{\eta}(\eta-s)^{\alpha-1} d s \\
& \left.+\frac{T+\eta}{(v+\alpha-1) T^{\nu} \Gamma(\alpha-1)|\Omega|} \int_{0}^{T}(T-s)^{v+\alpha-1} d s\right\} \\
\leq & (L r+M) T^{\alpha}\left(\frac{1}{\Gamma(\alpha+1)}+\frac{\eta^{2}(v+2(\alpha-1))}{(v+\alpha-1) T \Gamma(\alpha+1)|\Omega|}\right. \\
& \left.+\frac{T+\eta}{(v+\alpha-1)(v+\alpha) \Gamma(\alpha-1)|\Omega|}\right) \\
= & (L r+M) \frac{\Lambda}{L} \leq r,
\end{aligned}
$$

which proves that $A B_{r} \subset B_{r}$.

Now let $u, v \in \mathcal{C}$. Then, for $t \in[0, T]$, we have

$$
\begin{aligned}
|(A u)(t)-(A v)(t)| & \int_{0}^{t} \frac{(t-s)^{\alpha-1}}{\Gamma(\alpha)}|f(s, u(s))-f(s, v(s))| d s \\
& +\frac{T^{\alpha-1}(v+2(\alpha-1))}{(v+\alpha-1) \eta^{\alpha-2} \Gamma(\alpha)|\Omega|} \int_{0}^{\eta}(\eta-s)^{\alpha-1}|f(s, u(s))-f(s, v(s))| d s \\
& +\frac{T^{\alpha-2}(T+\eta)}{(v+\alpha-1) T^{v+\alpha-2} \Gamma(\alpha-1)|\Omega|} \\
& \times \int_{0}^{T}(T-s)^{v+\alpha-1}|f(s, u(s))-f(s, v(s))| d s \\
\leq & L \int_{0}^{t} \frac{(t-s)^{\alpha-1}}{\Gamma(\alpha)}|u(s)-v(s)| d s \\
& +\frac{L T^{\alpha-1}(v+2(\alpha-1))}{(v+\alpha-1) \eta^{\alpha-2} \Gamma(\alpha)|\Omega|} \int_{0}^{\eta}(\eta-s)^{\alpha-1}|u(s)-v(s)| d s \\
& +\frac{L(T+\eta)}{(v+\alpha-1) T^{v} \Gamma(\alpha-1)|\Omega|} \int_{0}^{T}(T-s)^{v+\alpha-1}|u(s)-v(s)| d s \\
\leq & \frac{L T^{\alpha}}{\Gamma(\alpha+1)}\|u-v\|+\frac{L \eta^{2} T^{\alpha-1}(v+2(\alpha-1))}{(v+\alpha-1) \Gamma(\alpha+1)|\Omega|}\|u-v\| \\
& +\frac{L T^{\alpha}(T+\eta)}{(v+\alpha-1)(v+\alpha) \Gamma(\alpha-1)|\Omega|}\|u-v\|
\end{aligned}
$$




$$
\begin{aligned}
= & L T^{\alpha}\left\{\frac{1}{\Gamma(\alpha+1)}+\frac{\eta^{2}(v+2(\alpha-1))}{(v+\alpha-1) T \Gamma(\alpha+1)|\Omega|}\right. \\
& \left.+\frac{T+\eta}{(v+\alpha-1)(v+\alpha) \Gamma(\alpha-1)|\Omega|}\right\}\|u-v\| .
\end{aligned}
$$

Therefore,

$$
\|A u-A v\| \leq \Lambda\|u-v\| .
$$

From (3.1), $A$ is a contraction. As a consequence of Banach's fixed point theorem, we get that $A$ has a fixed point which is a unique solution of problem (1.6)-(1.7).

Now we give another existence and uniqueness result for BVP (1.6)-(1.7) by using Banach's fixed point theorem and Hölder's inequality.

Theorem 3.2 Suppose that the continuous function $f$ satisfies the following assumption:

$\left(\mathrm{H}_{2}\right)\left|f\left(t, x_{1}\right)-f\left(t, x_{2}\right)\right| \leq m(t)\left|x_{1}-x_{2}\right|$, for $t \in[0, T], x_{i} \in \mathbb{R}, i=1,2$ and $m \in L^{\frac{1}{\gamma}}\left([0, T], \mathbb{R}^{+}\right)$, $\gamma \in(0,1)$.

Denote $\|m\|=\left(\int_{0}^{T}|m(s)|^{\frac{1}{\gamma}} d s\right)^{\gamma}$.If

$$
\begin{aligned}
& \frac{\|m\|}{\Gamma(\alpha)}\left\{T^{\alpha-\gamma}\left(\frac{1-\gamma}{\alpha-\gamma}\right)^{1-\gamma}+\frac{T^{\alpha-1} \eta^{2-\gamma}(v+2(\alpha-1))}{(v+\alpha-1)|\Omega|}\left(\frac{1-\gamma}{\alpha-\gamma}\right)^{1-\gamma}\right. \\
& \left.\quad+\frac{T^{\alpha-\gamma}(T+\eta)(\alpha-1)}{(v+\alpha-1)|\Omega|}\left(\frac{1-\gamma}{v+\alpha-\gamma}\right)^{1-\gamma}\right\}<1,
\end{aligned}
$$

then boundary value problem (1.6)-(1.7) has a unique solution.

Proof For $u, v \in \mathcal{C}$ and for each $t \in[0, T]$, by Hölder's inequality, we have

$$
\begin{aligned}
|(A u)(t)-(A v)(t)| & \\
\leq & \int_{0}^{t} \frac{(t-s)^{\alpha-1}}{\Gamma(\alpha)}|f(s, u(s))-f(s, v(s))| d s \\
& +\frac{T^{\alpha-1}(v+2(\alpha-1))}{(v+\alpha-1) \eta^{\alpha-2} \Gamma(\alpha)|\Omega|} \int_{0}^{\eta}(\eta-s)^{\alpha-1}|f(s, u(s))-f(s, v(s))| d s \\
& +\frac{T^{\alpha-2}(T+\eta)}{(v+\alpha-1) T^{v+\alpha-2} \Gamma(\alpha-1)|\Omega|} \int_{0}^{T}(T-s)^{\nu+\alpha-1}|f(s, u(s))-f(s, v(s))| d s \\
\leq & \int_{0}^{t} \frac{(t-s)^{\alpha-1}}{\Gamma(\alpha)} m(s)|u(s)-v(s)| d s \\
& +\frac{T^{\alpha-1}(v+2(\alpha-1))}{(v+\alpha-1) \eta^{\alpha-2} \Gamma(\alpha)|\Omega|} \int_{0}^{\eta}(\eta-s)^{\alpha-1} m(s)|u(s)-v(s)| d s \\
& +\frac{T^{\alpha-2}(T+\eta)}{(v+\alpha-1) T^{v+\alpha-2} \Gamma(\alpha-1)|\Omega|} \int_{0}^{T}(T-s)^{\nu+\alpha-1} m(s)|u(s)-v(s)| d s \\
\leq & \frac{1}{\Gamma(\alpha)}\left(\int_{0}^{t}\left((t-s)^{\alpha-1}\right)^{\frac{1}{1-\gamma}} d s\right)^{1-\gamma}\left(\int_{0}^{t}(m(s))^{1 / \gamma} d s\right)^{\gamma}\|u-v\|
\end{aligned}
$$




$$
\begin{aligned}
& +\frac{T^{\alpha-1}(v+2(\alpha-1))}{(v+\alpha-1) \eta^{\alpha-2}|\Omega| \Gamma(\alpha)} \\
& \times\left(\int_{0}^{\eta}\left((\eta-s)^{\alpha-1}\right)^{\frac{1}{1-\gamma}} d s\right)^{1-\gamma}\left(\int_{0}^{\eta}(m(s))^{1 / \gamma} d s\right)^{\gamma}\|u-v\| \\
& +\frac{T+\eta}{(v+\alpha-1) T^{v} \Gamma(\alpha-1)|\Omega|} \\
& \times\left(\int_{0}^{T}\left((T-s)^{v+\alpha-1}\right)^{\frac{1}{1-\gamma}} d s\right)^{1-\gamma}\left(\int_{0}^{T}(m(s))^{1 / \gamma} d s\right)^{\gamma}\|u-v\| \\
& \leq \frac{\|m\|}{\Gamma(\alpha)}\left\{T^{\alpha-\gamma}\left(\frac{1-\gamma}{\alpha-\gamma}\right)^{1-\gamma}+\frac{T^{\alpha-1} \eta^{2-\gamma}(v+2(\alpha-1))}{(v+\alpha-1)|\Omega|}\left(\frac{1-\gamma}{\alpha-\gamma}\right)^{1-\gamma}\right. \\
& \left.+\frac{T^{\alpha-\gamma}(T+\eta)(\alpha-1)}{(v+\alpha-1)|\Omega|}\left(\frac{1-\gamma}{v+\alpha-\gamma}\right)^{1-\gamma}\right\}\|u-v\| .
\end{aligned}
$$

It follows that $A$ is a contraction mapping. Hence Banach's fixed point theorem implies that $A$ has a unique fixed point which is the unique solution of problem (1.6)-(1.7). This completes the proof.

\subsection{Existence result via Krasnoselskii's fixed point theorem}

Lemma 3.1 (Krasnoselskii's fixed point theorem) [21] Let $M$ be a closed, bounded, convex and nonempty subset of a Banach space X. Let $A, B$ be the operators such that (a) $A x+B y \in$ $M$ whenever $x, y \in M$; (b) $A$ is compact and continuous; (c) $B$ is a contraction mapping. Then there exists $z \in M$ such that $z=A z+B z$.

Theorem 3.3 Let $f:[0, T] \times \mathbb{R} \rightarrow \mathbb{R}$ be a continuous function satisfying $\left(\mathrm{H}_{1}\right)$. Moreover, we assume that

$\left(\mathrm{H}_{3}\right)|f(t, u)| \leq \mu(t), \forall(t, u) \in[0, T] \times \mathbb{R}$, and $\mu \in C\left([0, T], \mathbb{R}^{+}\right)$.

Then boundary value problem (1.6)-(1.7) has at least one solution on $[0, T]$ if

$$
L T^{\alpha}\left\{\frac{\eta^{2}(v+2(\alpha-1))}{(v+\alpha-1) T \Gamma(\alpha+1)|\Omega|}+\frac{T+\eta}{(\nu+\alpha-1)(v+\alpha) \Gamma(\alpha-1)|\Omega|}\right\}<1 .
$$

Proof Letting $\sup _{t \in[0, T]}|\mu(t)|=\|\mu\|$, we fix

$$
\bar{r} \geq T^{\alpha}\|\mu\|\left\{\frac{1}{\Gamma(\alpha+1)}+\frac{\eta^{2}(v+2(\alpha-1))}{(v+\alpha-1) T \Gamma(\alpha+1)|\Omega|}+\frac{T+\eta}{(v+\alpha-1)(v+\alpha) \Gamma(\alpha-1)|\Omega|}\right\}
$$

and consider $B_{\bar{r}}=\{u \in \mathcal{C}:\|u\| \leq \bar{r}\}$. We define the operators $\mathcal{P}$ and $\mathcal{Q}$ on $B_{\bar{r}}$ as

$$
\begin{aligned}
(\mathcal{P} u)(t)= & \int_{0}^{t} \frac{(t-s)^{\alpha-1}}{\Gamma(\alpha)} f(s, u(s)) d s, \\
(\mathcal{Q} u)(t)= & -\frac{(v+\alpha-1) t^{\alpha-1}-T(\alpha-1) t^{\alpha-2}}{(v+\alpha-1) \eta^{\alpha-2} \Gamma(\alpha) \Omega} \int_{0}^{\eta}(\eta-s)^{\alpha-1} f(s, u(s)) d s \\
& +\frac{t^{\alpha-1}-\eta t^{\alpha-2}}{(v+\alpha-1) T^{\nu+\alpha-2} \Gamma(\alpha-1) \Omega} \int_{0}^{T}(T-s)^{v+\alpha-1} f(s, u(s)) d s .
\end{aligned}
$$


For $u, v \in B_{\bar{r}}$, we find that

$$
\begin{aligned}
& \|\mathcal{P} u+\mathcal{Q} v\| \\
& \quad \leq T^{\alpha}\|\mu\|\left\{\frac{1}{\Gamma(\alpha+1)}+\frac{\eta^{2}(v+2(\alpha-1))}{(v+\alpha-1) T \Gamma(\alpha+1)|\Omega|}+\frac{T+\eta}{(v+\alpha-1)(v+\alpha) \Gamma(\alpha-1)|\Omega|}\right\} \\
& \quad \leq \bar{r} .
\end{aligned}
$$

Thus, $\mathcal{P} u+\mathcal{Q} v \in B_{\bar{r}}$. It follows from assumption $\left(\mathrm{H}_{3}\right)$ together with (3.2) that $\mathcal{Q}$ is a contraction mapping. Continuity of $f$ implies that the operator $\mathcal{P}$ is continuous. Also, $\mathcal{P}$ is uniformly bounded on $B_{\bar{r}}$ as

$$
\|\mathcal{P} u\| \leq \frac{T^{\alpha}}{\Gamma(\alpha+1)}\|\mu\| .
$$

Now we prove the compactness of the operator $\mathcal{P}$.

We define $\sup _{(t, u) \in[0, T] \times B_{\bar{r}}}|f(t, u)|=\bar{f}<\infty$, and consequently we have

$$
\begin{aligned}
\left|(\mathcal{P} u)\left(t_{2}\right)-(\mathcal{P} u)\left(t_{1}\right)\right|= & \frac{1}{\Gamma(\alpha)} \mid \int_{0}^{t_{1}}\left[\left(t_{2}-s\right)^{\alpha-1}-\left(t_{1}-s\right)^{\alpha-1}\right] f(s, u(s)) d s \\
& +\int_{t_{1}}^{t_{2}}\left(t_{2}-s\right)^{\alpha-1} f(s, u(s)) d s \mid \\
\leq & \frac{\bar{f}}{\Gamma(\alpha+1)}\left|t_{1}^{\alpha}-t_{2}^{\alpha}\right|,
\end{aligned}
$$

which is independent of $u$. Thus, $\mathcal{P}$ is equicontinuous. So $\mathcal{P}$ is relatively compact on $B_{\bar{r}}$. Hence, by the Arzelá-Ascoli theorem, $\mathcal{P}$ is compact on $B_{\bar{r}}$. Thus all the assumptions of Lemma 3.1 are satisfied. So the conclusion of Lemma 3.1 implies that boundary value problem (1.6)-(1.7) has at least one solution on $[0, T]$.

\subsection{Existence result via Leray-Schauder's nonlinear alternative}

Theorem 3.4 (Nonlinear alternative for single-valued maps) [22] Let E be a Banach space, $C$ be a closed, convex subset of $E, U$ be an open subset of $C$ and $0 \in U$. Suppose that $F: \bar{U} \rightarrow$ $C$ is a continuous, compact (that is, $F(\bar{U})$ is a relatively compact subset of $C$ ) map. Then either

(i) F has a fixed point in $\bar{U}$, or

(ii) there is a $u \in \partial U$ (the boundary of $U$ in $C$ ) and $\lambda \in(0,1)$ with $u=\lambda F(u)$.

Theorem 3.5 Assume that

$\left(\mathrm{H}_{4}\right)$ there exists a continuous nondecreasing function $\psi:[0, \infty) \rightarrow(0, \infty)$ and a function $p \in L^{1}\left([0, T], \mathbb{R}^{+}\right)$such that

$$
|f(t, u)| \leq p(t) \psi(\|u\|) \quad \text { for each }(t, u) \in[0, T] \times \mathbb{R}
$$

$\left(\mathrm{H}_{5}\right)$ there exists a constant $M>0$ such that

$$
\frac{M}{T^{\alpha} \psi(M)\|p\|_{L^{1}\left\{\frac{1}{\Gamma(\alpha+1)}+\frac{\eta^{2}(v+2(\alpha-1))}{(v+\alpha-1) T \Gamma(\alpha+1) \Omega}+\frac{T+\eta}{(v+\alpha-1)(v+\alpha) \Gamma(\alpha-1) \Omega}\right\}}}>1 .
$$

Then boundary value problem (1.6)-(1.7) has at least one solution on $[0, T]$. 
Proof We show that $A$ maps bounded sets (balls) into bounded sets in $C([0, T], \mathbb{R})$. For a positive number $\rho$, let $B_{\rho}=\{u \in C([0, T], \mathbb{R}):\|u\| \leq \rho\}$ be a bounded ball in $C([0, T], \mathbb{R})$. Then for $t \in[0, T]$ we have

$$
\begin{aligned}
& |(A u)(t)| \leq \frac{1}{\Gamma(\alpha)} \int_{0}^{t}(t-s)^{\alpha-1}|f(s, u(s))| d s \\
& +\frac{T^{\alpha-1}(v+2(\alpha-1))}{(v+\alpha-1) \eta^{\alpha-2} \Gamma(\alpha)|\Omega|} \int_{0}^{\eta}(\eta-s)^{\alpha-1}|f(s, u(s))| d s \\
& +\frac{T^{\alpha-2}(T+\eta)}{(\nu+\alpha-1) T^{\nu+\alpha-2} \Gamma(\alpha-1)|\Omega|} \int_{0}^{T}(T-s)^{\nu+\alpha-1}|f(s, u(s))| d s \\
& \leq \frac{\psi(\|u\|)}{\Gamma(\alpha)} \int_{0}^{t}(t-s)^{\alpha-1} p(s) d s \\
& +\frac{\psi(\|u\|) T^{\alpha-1}(v+2(\alpha-1))}{(v+\alpha-1) \eta^{\alpha-2} \Gamma(\alpha)|\Omega|} \int_{0}^{\eta}(\eta-s)^{\alpha-1} p(s) d s \\
& +\frac{\psi(\|u\|)(T+\eta)}{(v+\alpha-1) T^{\nu} \Gamma(\alpha-1)|\Omega|} \int_{0}^{T}(T-s)^{\nu+\alpha-1} p(s) d s \\
& \leq \frac{\psi(\|u\|)\|p\|_{L^{1}}}{\Gamma(\alpha)} \int_{0}^{T}(T-s)^{\alpha-1} d s \\
& +\frac{\psi(\|u\|)\|p\|_{L^{1}} T^{\alpha-1}(v+2(\alpha-1))}{(v+\alpha-1) \eta^{\alpha-2} \Gamma(\alpha)|\Omega|} \int_{0}^{\eta}(\eta-s)^{\alpha-1} d s \\
& +\frac{\psi(\|u\|)\|p\|_{L^{1}}(T+\eta)}{(v+\alpha-1) T^{\nu} \Gamma(\alpha-1)|\Omega|} \int_{0}^{T}(T-s)^{\nu+\alpha-1} d s \\
& \leq \frac{T^{\alpha} \psi(\|u\|)\|p\|_{L^{1}}}{\Gamma(\alpha+1)}+\frac{\eta^{2} \psi(\|u\|)\|p\|_{L^{1}} T^{\alpha-1}(v+2(\alpha-1))}{(v+\alpha-1) \Gamma(\alpha+1)|\Omega|} \\
& +\frac{T^{\alpha} \psi(\|u\|)\|p\|_{L^{1}}(T+\eta)}{(v+\alpha-1)(v+\alpha) \Gamma(\alpha-1)|\Omega|} \\
& =T^{\alpha} \psi(\|u\|)\|p\|_{L^{1}}\left\{\frac{1}{\Gamma(\alpha+1)}+\frac{\eta^{2}(v+2(\alpha-1))}{(v+\alpha-1) T \Gamma(\alpha+1)|\Omega|}\right. \\
& \left.+\frac{T+\eta}{(v+\alpha-1)(v+\alpha) \Gamma(\alpha-1)|\Omega|}\right\} \text {. }
\end{aligned}
$$

Consequently,

$$
\begin{aligned}
\|A u\| \leq & T^{\alpha} \psi(\rho)\|p\|_{L^{1}}\left\{\frac{1}{\Gamma(\alpha+1)}+\frac{\eta^{2}(v+2(\alpha-1))}{(v+\alpha-1) T \Gamma(\alpha+1)|\Omega|}\right. \\
& \left.+\frac{T+\eta}{(v+\alpha-1)(v+\alpha) \Gamma(\alpha-1)|\Omega|}\right\} .
\end{aligned}
$$

Next we show that $A$ maps bounded sets into equicontinuous sets of $C([0, T], \mathbb{R})$. Let $t_{1}, t_{2} \in[0, T]$ with $t_{1}<t_{2}$ and $u \in B_{\rho}$. Then we have

$$
\begin{aligned}
& \left|(A u)\left(t_{2}\right)-(A u)\left(t_{1}\right)\right| \\
& \quad \leq\left|\frac{1}{\Gamma(\alpha)} \int_{0}^{t_{2}}\left(t_{2}-s\right)^{\alpha-1}\right| f(s, u(s))\left|d s-\frac{1}{\Gamma(\alpha)} \int_{0}^{t_{1}}\left(t_{1}-s\right)^{\alpha-1}\right| f(s, u(s))|d s|
\end{aligned}
$$




$$
\begin{aligned}
& +\frac{(v+\alpha-1)\left|t_{2}^{\alpha-1}-t_{1}^{\alpha-1}\right|+T(\alpha-1)\left|t_{2}^{\alpha-2}-t_{1}^{\alpha-2}\right|}{(v+\alpha-1) \eta^{\alpha-2} \Gamma(\alpha)|\Omega|} \int_{0}^{\eta}(\eta-s)^{\alpha-1}|f(s, u(s))| d s \\
& +\frac{\eta\left|t_{1}^{\alpha-2}-t_{1}^{\alpha-2}\right|+\left|t_{2}^{\alpha-1}-t_{1}^{\alpha-1}\right|}{(v+\alpha-1) T^{\nu+\alpha-2} \Gamma(\alpha-1)|\Omega|} \int_{0}^{T}(T-s)^{\nu+\alpha-1}|f(s, u(s))| d s \\
& \leq\left|\frac{1}{\Gamma(\alpha)} \int_{0}^{t_{2}}\left(t_{2}-s\right)^{\alpha-1} p(s) \psi(\rho) d s-\frac{1}{\Gamma(\alpha)} \int_{0}^{t_{1}}\left(t_{1}-s\right)^{\alpha-1} p(s) \psi(\rho) d s\right| \\
& +\frac{(v+\alpha-1)\left|t_{2}^{\alpha-1}-t_{1}^{\alpha-1}\right|+T(\alpha-1)\left|t_{2}^{\alpha-2}-t_{1}^{\alpha-2}\right|}{(v+\alpha-1) \eta^{\alpha-2} \Gamma(\alpha)|\Omega|} \int_{0}^{\eta}(\eta-s)^{\alpha-1} p(s) \psi(\rho) d s \\
& +\frac{\eta\left|t_{1}^{\alpha-2}-t_{1}^{\alpha-2}\right|+\left|t_{2}^{\alpha-1}-t_{1}^{\alpha-1}\right|}{(v+\alpha-1) T^{v+\alpha-2} \Gamma(\alpha-1)|\Omega|} \int_{0}^{T}(T-s)^{\nu+\alpha-1} p(s) \psi(\rho) d s .
\end{aligned}
$$

Obviously the right-hand side of the above inequality tends to zero independently of $x \in B_{\rho}$ as $t_{2}-t_{1} \rightarrow 0$. As $A$ satisfies the above assumptions, therefore it follows by the Arzelá-Ascoli theorem that $A: C([0, T], \mathbb{R}) \rightarrow C([0, T], \mathbb{R})$ is completely continuous.

Let $u$ be a solution. Then, for $t \in[0, T]$, and following similar computations as in the first step, we have

$$
\begin{aligned}
|u(t)| \leq & T^{\alpha} \psi(\|u\|)\|p\|_{L^{1}}\left\{\frac{1}{\Gamma(\alpha+1)}+\frac{\eta^{2}(v+2(\alpha-1))}{(v+\alpha-1) T \Gamma(\alpha+1)|\Omega|}\right. \\
& \left.+\frac{T+\eta}{(v+\alpha-1)(v+\alpha) \Gamma(\alpha-1)|\Omega|}\right\} .
\end{aligned}
$$

Consequently, we have

$$
\frac{\|u\|}{T^{\alpha} \psi(\|u\|)\|p\|_{L^{1}}\left\{\frac{1}{\Gamma(\alpha+1)}+\frac{\eta^{2}(v+2(\alpha-1))}{(v+\alpha-1) T \Gamma(\alpha+1)|\Omega|}+\frac{T+\eta}{(v+\alpha-1)(v+\alpha) \Gamma(\alpha-1)|\Omega|}\right\}} \leq 1 .
$$

In view of $\left(\mathrm{H}_{5}\right)$, there exists $M$ such that $\|u\| \neq M$. Let us set

$$
U=\{u \in C([0, T], \mathbb{R}):\|u\|<M\}
$$

Note that the operator $A: \bar{U} \rightarrow C([0, T], \mathbb{R})$ is continuous and completely continuous. From the choice of $U$, there is no $u \in \partial U$ such that $u=\lambda A u$ for some $\lambda \in(0,1)$. Consequently, by the nonlinear alternative of Leray-Schauder type (Theorem 3.4), we deduce that $A$ has a fixed point $u \in \bar{U}$ which is a solution of problem (1.6)-(1.7). This completes the proof.

\section{Examples}

Example 4.1 Consider the following fractional integral boundary value problem:

$$
\begin{aligned}
& D^{\frac{3}{2}} u(t)=\frac{e^{-t}}{4\left(1+e^{t}\right)} \cdot \frac{|u(t)|}{1+|u(t)|}, \quad t \in\left(0, \frac{8}{3}\right), \\
& u\left(\frac{5}{3}\right)=0, \quad I^{\frac{7}{2}} u\left(\frac{8}{3}\right)=0 .
\end{aligned}
$$

Here $\alpha=3 / 2, v=7 / 2, \eta=5 / 3, T=8 / 3$ and $f(t, u)=\left(e^{-t} / 4\left(1+e^{t}\right)\right)(|u| /(1+|u|))$ and $\Omega=$ $\eta-(\alpha-1) T /(v+\alpha-1)=4 / 3 \neq 0$. Since $|f(t, u)-f(t, v)| \leq(1 / 8)|u-v|$, then $\left(\mathrm{H}_{1}\right)$ is satisfied 
with $L=1 / 8$. We can show that

$$
\begin{aligned}
& L T^{\alpha}\left\{\frac{1}{\Gamma(\alpha+1)}+\frac{\eta^{2}[v+2(\alpha-1)]}{(v+\alpha-1) T \Gamma(\alpha+1)|\Omega|}+\frac{\eta+T}{(v+\alpha-1)(v+\alpha) \Gamma(\alpha-1)|\Omega|}\right\} \\
& \quad=\frac{1}{8}\left(\frac{8}{3}\right)^{\frac{3}{2}}\left[\frac{4}{3 \sqrt{\pi}}+\frac{75}{64 \sqrt{\pi}}+\frac{13}{80 \sqrt{\pi}}\right] \approx 0.819268997<1 .
\end{aligned}
$$

Hence, by Theorem 3.1, boundary value problem (4.1)-(4.2) has a unique solution on $[0,8 / 3]$.

Example 4.2 Consider the following fractional integral boundary value problem:

$$
\begin{aligned}
& D^{\frac{5}{3}} x(t)=t^{2} \tan ^{-1} x(t), \quad t \in\left(0, \frac{1}{2}\right), \\
& x\left(\frac{3}{8}\right)=0, \quad I^{\frac{2}{3}} x\left(\frac{1}{2}\right)=0 .
\end{aligned}
$$

Set $\alpha=5 / 3, v=2 / 3, \eta=3 / 8, T=1 / 2, f(t, x)=t^{2} \tan ^{-1} x$ and choose $\gamma=(1 / 12) \in(0,1)$. It is easy to see that $\Omega=\eta-(\alpha-1) T /(v+\alpha-1)=1 / 8 \neq 0$. Since $|f(t, x)-f(t, y)|=t^{2} \mid \tan ^{-1} x-$ $\tan ^{-1} y\left|\leq t^{2}\right| x-y \mid$, then $\left(\mathrm{H}_{2}\right)$ is satisfied with $m(t)=t^{2} \in L^{12}\left([0,1 / 2], \mathbb{R}^{+}\right)$. We can show that

$$
\|m\|=\left(\int_{0}^{\frac{1}{2}}\left|s^{2}\right|^{12} d s\right)^{\frac{1}{12}} \approx 0.1804509
$$

and

$$
\begin{gathered}
\frac{\|m\|}{\Gamma(\alpha)}\left\{T^{\alpha-\gamma}\left(\frac{1-\gamma}{\alpha-\gamma}\right)^{1-\gamma}+\frac{\eta^{2-\gamma} T^{\alpha-1}[v+2(\alpha-1)]}{(v+\alpha-1)|\Omega|}\left(\frac{1-\gamma}{\alpha-\gamma}\right)^{1-\gamma}\right. \\
\left.+\frac{T^{\alpha-\gamma}(\eta+T)(\alpha-1)}{(v+\alpha-1)|\Omega|}\left(\frac{1-\gamma}{v+\alpha-\gamma}\right)^{1-\gamma}\right\} \approx 0.2826838<1 .
\end{gathered}
$$

Hence, by Theorem 3.2, boundary value problem (4.3)-(4.4) has a unique solution on $[0,1 / 2]$.

Example 4.3 Consider the following fractional integral boundary value problem:

$$
\begin{aligned}
& D^{\frac{9}{5}} x(t)=\frac{t}{10 \pi} \sin (\pi x)+\frac{(t+1) x^{2}}{1+x^{2}}, \quad t \in\left(0, \frac{3}{4}\right), \\
& x\left(\frac{1}{2}\right)=0, \quad I^{\frac{4}{5}} x\left(\frac{3}{4}\right)=0 .
\end{aligned}
$$

Set $\alpha=9 / 5, v=4 / 5, \eta=1 / 2, T=3 / 4, f(t, x)=(t / 10 \pi) \sin (\pi x)+\left((t+1) x^{2} /\left(1+x^{2}\right)\right)$. It is easy to see that $\Omega=\eta-(\alpha-1) T /(\nu+\alpha-1)=1 / 8 \neq 0$. Clearly,

$$
|f(t, x)|=\left|\frac{t}{10 \pi} \sin (\pi x)+\frac{(t+1) x^{2}}{1+x^{2}}\right| \leq(t+1)\left(\frac{\|x\|}{10}+1\right) .
$$


Choosing $p(t)=t+1, \psi(\|x\|)=(\|x\| / 10)+1$, we obtain

$$
\frac{M}{T^{\alpha}\|p\|_{L^{1}}\left\{\frac{1}{\Gamma(\alpha+1)}+\frac{\eta^{2}[v+2(\alpha-1)]}{(v+\alpha-1) T \Gamma(\alpha+1)|\Omega|}+\frac{T+\eta}{(v+\alpha-1)(v+\alpha) \Gamma(\alpha-1)|\Omega|}\right\}}>\frac{M}{10}+1,
$$

which implies that $M>4.495162241$. Hence, by Theorem 3.5, boundary value problem (4.5)-(4.6) has at least one solution on $[0,3 / 4]$.

\section{Competing interests}

The authors declare that they have no competing interests.

\section{Authors' contributions}

All authors contributed equally in this article. They read and approved the final manuscript

\section{Author details}

${ }^{1}$ Department of Mathematics, Faculty of Applied Science, King Mongkut's University of Technology North Bangkok, Bangkok, 10800, Thailand. ²Department of Mathematics, University of loannina, loannina, 451 10, Greece.

\section{Authors' information}

Sotiris K Ntouyas is a member of Nonlinear Analysis and Applied Mathematics (NAAM) - Research Group at King Abdulaziz University, Jeddah, Saudi Arabia.

\section{Acknowledgements}

The present paper was done while J Tariboon and T Sitthiwirattham visited the Department of Mathematics of the University of loannina, Greece. It is a pleasure for them to thank Professor SK Ntouyas for his warm hospitality. This research of J Tariboon and T Sitthiwirattham is supported by King Mongkut's University of Technology North Bangkok, Thailand

Received: 22 April 2013 Accepted: 28 June 2013 Published: 13 July 2013

\section{References}

1. Miller, KS, Ross, B: An Introduction to the Fractional Calculus and Differential Equations. Wiley, New York (1993)

2. Samko, SG, Kilbas, AA, Marichev, Ol: Fractional Integrals and Derivatives: Theory and Applications. Gordon \& Breach, Yverdon (1993)

3. Podlubny, I: Fractional Differential Equations. Academic Press, San Diego (1999)

4. Kilbas, AA, Srivastava, HM, Trujillo, Jj: Theory and Applications of Fractional Differential Equations. North-Holland Mathematics Studies, vol. 204. Elsevier, Amsterdam (2006)

5. Lakshmikantham, V, Leela, S, Vasundhara Devi, J: Theory of Fractional Dynamic Systems. Cambridge Academic Publishers, Cambridge (2009)

6. Baleanu, D, Diethelm, K, Scalas, E, Trujillo, JJ: Fractional Calculus Models and Numerical Methods. Series on Complexity, Nonlinearity and Chaos. World Scientific, Boston (2012)

7. Diethelm, K: The Analysis of Fractional Differential Equations. An Application-Oriented Exposition Using Differential Operators of Caputo Type. Lecture Notes in Mathematics, vol. 2004. Springer, Berlin (2010)

8. Guezane-Lakoud, A, Khaldi, R: Positive solution to a higher order fractional boundary value problem with fractional integral condition. Rom. J. Math. Comput. Sci. 2, 41-54 (2012)

9. Kaufmann, E: Existence and nonexistence of positive solutions for a nonlinear fractional boundary value problem. Discrete Contin. Dyn. Syst. 2009, suppl., 416-423 (2009)

10. Wang, J, Xiang, H, Liu, Z: Positive solution to nonzero boundary values problem for a coupled system of nonlinear fractional differential equations. Int. J. Differ. Equ. 2010, Article ID 186928 (2010)

11. Bai, Z: On positive solutions of a nonlocal fractional boundary value problem. Nonlinear Anal. 72, $916-924$ (2010)

12. Sudsutad, W, Tariboon, J: Boundary value problems for fractional differential equations with three-point fractional integral boundary conditions. Adv. Differ. Equ. 2012, 93 (2012)

13. Ntouyas, SK: Existence results for nonlocal boundary value problems for fractional differential equations and inclusions with fractional integral boundary conditions. Discuss. Math., Differ. Incl. Control Optim. 33, 17-39 (2013)

14. Ntouyas, SK: Boundary value problems for nonlinear fractional differential equations and inclusions with nonlocal and fractional integral boundary conditions. Opusc. Math. 33, 117-138 (2013)

15. Guezane-Lakoud, A, Khaldi, R: Solvability of a fractional boundary value problem with fractional integral condition. Nonlinear Anal. 75, 2692-2700 (2012)

16. Ahmad, B, Ntouyas, SK, Assolani, A: Caputo type fractional differential equations with nonlocal Riemann-Liouville integral boundary conditions. J. Appl. Math. Comput. (2012). doi:10.1007/s12190-012-0610-8

17. Baleanu, D, Mustafa, OG, Agarwal, RP: An existence result for a superlinear fractional differential equation. Appl. Math. Lett. 23, 1129-1132 (2010)

18. Debbouche, A, Baleanu, D, Agarwal, RP: Nonlocal nonlinear integrodifferential equations of fractional orders. Bound. Value Probl. 2012, 78 (2012)

19. Nyamoradi, N, Baleanu, D, Agarwal, RP: On a multipoint boundary value problem for a fractional order differential inclusion on an infinite interval. Adv. Math. Phys. 2013, Article ID 823961 (2013)

20. Guezane-Lakoud, A, Khaldi, R: Solvability of a three-point fractional nonlinear boundary value problem. Differ. Equ. Dyn. Syst. 20, 395-403 (2012) 
21. Krasnoselskii, MA: Two remarks on the method of successive approximations. Usp. Mat. Nauk 10, 123-127 (1955)

22. Granas, A, Dugundji, J: Fixed Point Theory. Springer, New York (2003)

doi:10.1186/1687-1847-2013-213

Cite this article as: Tariboon et al.: Boundary value problems for a new class of three-point nonlocal

Riemann-Liouville integral boundary conditions. Advances in Difference Equations 2013 2013:213.

Submit your manuscript to a SpringerOpen ${ }^{\circ}$ journal and benefit from:

- Convenient online submission

- Rigorous peer review

- Immediate publication on acceptance

- Open access: articles freely available online

- High visibility within the field

- Retaining the copyright to your article

Submit your next manuscript at $>$ springeropen.com 